\title{
Structural Realism or Modal Empiricism?
}

\begin{abstract}
Structural realism has been suggested as the best compromise in the debate on scientific realism. It proposes that we should be realist about the relational structure of the world, not its nature. However it faces an important objection, first raised by Newman against Russell: if relations are not qualified, then the position is either trivial or collapses into empiricism, but if relations are too strongly qualified, then it is no longer structural realism. A way to overcome this difficulty is to talk about modal, or nomological relations instead of purely extensional relations. I argue that this is insufficient, for then, structural realism collapses into modal empiricism. I suggest, however, that modal empiricism could be the best position in the debate on scientific realism.
\end{abstract}

1 Objections to Structural Realism

2 How to Escape Newman's Objection?

3 Which Modal Relations are Retained in Theory Change?

4 Are Modal Relations Real?

5 Relativity and Fundamentality

6 Is Modal Empiricism Really Empiricism?

7 Could Modal Empiricism be the Best of Both Worlds? 
Scientific realism is a family of positions according to which our best scientific theories are true, or approximately true. Empiricism is a family of positions that puts emphasis on the central, perhaps exclusive role of experience in knowledge acquisition and justification. In the context of philosophy of science, empiricism can be understood in terms of epistemic commitments toward scientific theories, or, following van Fraassen ([1980])'s constructive empiricism, as a doctrine about the aim of science: according to constructive empiricism, this aim is to produce empirically adequate theories, that is to say, theories that 'save the phenomena', not true theories.

The epistemological debate between scientific realism and empiricism centres around two main arguments that push in opposite directions: the no-miracle argument and the pessimistic meta-induction. The no-miracle argument (Putnam [1975]) claims that scientific realism is the best (or only) explanation for the empirical success of scientific theories, or for the success of science in general in producing empirically successful theories (Psillos [1999], ch. 4). The argument puts emphasis on novel, unexpected predictions: it would be a miracle that the extension of theories to new domains of experience that they were not designed to account for proved successful if these theories were not true. The pessimistic meta-induction is an induction on past scientific theories, most of which are now abandoned, to the conclusion that contemporary theories are most probably false, contrarily to what the no-miracle argument suggests. They cannot be considered approximately true, because there is no ontological continuity between successive theories, and not even a continuity in the reference of theoretical terms, since many terms used in past theories, such as 'ether' or 'phlogiston', do not refer in light of contemporary theories (Laudan [1981]).

Worrall ([1989]) re-introduced structural realism (SR) as a solution of compromise in the debate between scientific realism and empiricism. SR is the position according to which we should only be realist about structure: the structure of our best theories corresponds to the structure of the world. Two versions of this position can be distinguished: an epistemic version, according to which we should remain agnostic about the nature of the world beyond its structure (we should not interpret theoretical terms, for example, we should not assume that they refer to natural properties), and an ontic version (French [1998]; Ladyman [1998]; Ladyman and Ross [2007]; French [2014]), according to which the nature of the world is structural: structure is all there is.

According to its proponents, SR can respond to the pessimistic meta-induction, because there is a continuity of structure between successive theories: the structure of abandoned theories is generally retained in theory change. It also does jus- 
tice to the no-miracle argument, because a correspondence of structure between our theories and the world is enough to explain their empirical success. However, there is an influential objection against SR, called Newman's objection, to the effect that SR would either be trivial or collapse into empiricism: it is merely committed to relations between our observations. If this is true, SR does not explain the empirical success of theories, but merely states that success, and therefore, it cannot account for novel predictions.

Some authors argue that talk of modal relations (or viewing the structure of the world as a nomological structure, rather than as an extensional structure) can help circumvent Newman's objection. In this article, I will argue that this move does not work: Newman's objection still applies to modal SR, because the modal relations that survive theory change cannot be 'real' relations that would explain the empirical success of theories: they are mere extensions of this empirical success to possible phenomena. Therefore, modal SR is nothing but empiricism-albeit modal empiricism (ME) ${ }^{1}$. Nevertheless, ME could be the best position of compromise in this debate, but not for the reasons structural realists are invoking.

I will first present the main objections against SR, including Newman's objection (section 1) and the different ways one could escape Newman's objection (section 2). Then I will examine to what extent introducing modalities is an acceptable solution: whether modal relations survive theory change (section 3), and whether the ones that do can explain empirical success (section 4) or correspond to the modal structure of reality (section 5). I will conclude that modal SR collapses into ME, and detail in which sense ME, although it is committed to natural modalities, is not a realist position (section 6). To finish, I will briefly examine how ME fares in the debate on scientific realism (section 7).

\section{Objections to Structural Realism}

Given that SR purports to answer two different arguments, the validity of which it assumes, objections against the position can be broadly classified into two types: the objections to the effect that SR cannot answer the pessimistic meta-induction any more than standard realism does (either because it is indistinct from standard

\footnotetext{
${ }^{1}$ I use the term in the sense given by Giere in (Churchland and Hooker [1985], ch. 4) as a position in epistemology of science. It should not be conflated with modal empiricism in general epistemology (Roca-Royes [2007]; Hanrahan [2009]), which concerns metaphysical modalities rather than nomological modalities, and which opposes modal rationalism rather than non-modal empiricism.
} 
realism, or because there is no structural continuity in theory change), and the objections to the effect that it cannot do justice to the no-miracle argument any more than empiricism does.

Regarding the first type of objection, Psillos ([1995]) claims that the nature versus structure dichotomy employed by structural realists is unclear. According to Psillos, standard realism has the resources to answer the pessimistic metainduction, but structural realism is not a consistent alternative since there is a continuum between nature and structure rather than a strict dichotomy. The structure of a theory informs us about the nature of the entities it posits (for example, how Newtonian mass relates to force and acceleration informs us about the nature of mass), and structure must be interpreted for a theory to make any prediction. In a similar vein, Papineau ([1996], p. 12) claims that a restriction of realism to structure is no restriction at all.

Another problem in this category, noted by Newman ([2005]), is that the structural realists must explicate what exactly structural continuity amounts to, beyond the intuitive idea that equations are somehow transposed from one theory to the other. Without any such account, they cannot claim to answer the pessimistic meta-induction argument. Some authors argue that there is no real continuity of structure between successive theories, apart from empirical structures. Psillos ([1999], ch. 7) claims that not all structure is retained in theory change. Stanford ([2003]) takes the example of Galton's law in biology, where the structural continuity is only empirical with Mendel's theory, but no relations between posited entities (here the genetic characteristics of ancestors of an organism) are retained. Redhead ([2001]) takes the example of the commutativity of observables in classical and quantum mechanics to argue that structural transformations are too important in theory change to really talk about structural continuity. Bueno ([2008]) gives other examples of structural loss in theory change. More recently, Pashby ([2012]) uses the example of the discovery of the positron to show how shifts in the metaphysical commitments of a theory may also be displayed in terms of changes in theoretical structure. This supports Psillos's contention that the nature-structure dichotomy is not so clear.

A common strategy for the structural realist facing this type of difficulty is to concentrate on well confirmed empirical relations between phenomena that are very unlikely to disappear in theory change. Thus, against Psillos's contention that structure must be interpreted to make predictions, Votsis ([2004]) notes that SR does not say that structure is not interpreted at all, but that it is only interpreted empirically. Structural realists can refer to the principle of correspondence (Post [1971], p. 228) for their purpose. According to this principle, 'any acceptable new 
theory $L$ should account for the success of its predecessor $S$ by "degenerating" into that theory under those conditions under which $S$ has been well confirmed by tests'. However, then, the ultimate benchmark for structural continuity between theories is their predictive success, and it is not so clear that anything is retained in theory change beyond empirical structures that are approximate and restricted to a limited domain of experience: an empiricist with structuralist leanings would readily agree.

This brings us to the second family of objections against SR: that SR does not say anything beyond empiricism. The most influential objection to that effect is dubbed Newman's objection, after Newman ([1928])'s argument against Russell ([1927])'s version of SR ${ }^{2}$. The argument can be expressed intuitively as follows: if relations are conceived of in purely extensional terms (a relation is defined by the objects it relates), then it suffices that some objects exist for all relations that could be defined between these objects to exist, mathematically speaking. So, for any mathematical structure to exist, it is sufficient that there exist enough objects in the world to bear that structure, and SR is a trivial position that is only committed to a cardinal claim (the existence of a certain number of objects in the world). To block that objection, the structuralist must say more, and qualify the kind of relations that she is talking about to differentiate them from purely mathematical relations, but then, the position is not purely structural: the structure must be interpreted.

As already noted, SR can accept that the structure be interpreted empirically. However, then, SR is only committed to a structure of observations (and a sufficient number of inaccessible objects to bear that structure), and the position is only superficially different from empiricism.

\section{How to Escape Newman's Objection?}

A useful framework to examine this objection is the Ramsey sentence formalism. It starts from the assumption that the cognitive content of a theory can be expressed as a logical sentence $\Pi$ in a vocabulary comprising observational terms $O_{i}$ and theoretical terms $T_{i}$.

$$
T=\prod\left(O_{1}, O_{2}, \ldots, O_{n}, T_{1}, T_{2}, \ldots, T_{m}\right)
$$

\footnotetext{
${ }^{2}$ The term 'structural realism' was coined later by Maxwell ([1971]), who took inspiration from Russell.
} 
The idea that theoretical terms should not be interpreted can be implemented by replacing theoretical terms by variables $t_{i}$ over which one quantifies existentially: this is the Ramsey sentence of the theory.

$$
\left(\exists t_{1}, t_{2}, \ldots, t_{m}\right) \prod\left(O_{1}, O_{2}, \ldots, O_{n}, t_{1}, t_{2}, \ldots, t_{m}\right)
$$

The difference with the former formulation is that while the $T_{i}$, as part of our vocabulary, are interpreted, the $t_{i}$ are not. According to Maxwell ([1971]), what SR is committed to can thus be expressed by 'rameseyfying' theories: only the observational terms are interpreted, but the structure of the theory is retained. In particular, one does not have to assume that the $t_{i}$ refer to natural properties: they could be multiply realisable in light of a new theory (French [2014], ch. 5.9). This is how SR, thus formulated, can answer the pessimistic meta-induction. But following Newman's objection, it cannot do justice to the no-miracle argument, because it does not explain observable regularities: it is a mere statement of these regularities, plus a cardinal claim.

In this framework, Newman's objection takes the form of a theorem of secondorder logic (Demopoulos and Friedman [1985]): the Ramsey sentence of a theory is true if and only if all the empirical consequences of the theory (the observational sentences that can be deduced from the theory) are true, and if there exist a certain number of objects.

Some authors, such as Worrall and Zahar ([2004]), claim that this is sufficient for structural realism: the Ramsey sentence expresses all the empirical consequences of a theory, for all past, present and future phenomena in the universe, in the most compact possible way, and this, in itself, is informative. However, constructive empiricism is also committed to our theories being empirically adequate for all past, present and future phenomena, and it's not clear what 'informative' means here. It is not even obvious that this compact formulation is retained in theory change (in particular if theoretical terms of past theories do not appear any more in new theories). If it is a way to claim that the properties and relations the $t_{i}$ refer to are 'natural' rather than fictitious, then we are back to standard realism.

Being a theorem of second-order logic, Newman's objection is inescapable if one assumes that a Ramsey sentence is the right way to express the content of a theory to which SR is committed. But perhaps this formalism unduly attributes implicit commitments to the structural realist. Among these implicit commitments are:

- the assumption of a dichotomy between observational and theoretical terms, 
- the assumption that a theory quantifies universally over objects, properties and relations,

- the assumption that the content of a theory is extensional.

Melia and Saatsi ([2006]) suggest different ways to depart from this formalism to escape Newman's objection by rejecting one or other of these assumptions ${ }^{3}$.

First, one can add mixed predicates to the vocabulary: predicates that apply indifferently to observable or unobservable objects (for example, an atom is smaller than a molecule just as a cat is smaller than a dog). This is the solution envisaged by Cruse $([2005])^{4}$. This constitutes an improvement, but a limited one: Newman's objection still applies to the purely theoretical content of theories.

Second, one can restrict the intended domain of the theory instead of assuming universal quantification. This can apply either to objects (for example, the theory applies only to concrete or physical objects, not all objects) or to properties and relations. The first option amounts to applying a new predicate to all objects concerned by the theory, but Newman's objection also applies to the new resulting theory, once ramseyfied, so this solution will not work. The second option amounts to qualifying properties and relations with a second-order predicate. In this context, the question is whether one could do so without falling prey to the pessimistic meta-induction. Melia and Saatsi consider different possibilities, and conclude that nothing really works. If one qualifies properties and relations as 'natural', then it seems that we are back to standard realism, and properties once deemed natural (such as 'being phlogiston') are now considered inexistent. But if one qualifies properties and relations as 'qualitative' (that is, as arbitrary combinations of natural properties and relations) then Newman's objection cannot be blocked: almost any structure can be realized by qualitative properties and relations.

\footnotetext{
${ }^{3}$ French and Ladyman ([2003]) suggested that Newman's objection is an artefact of the statement view of theories. The semantic view, by contrast, conceives of theories as collections of models rather than sentences. In this context, structural correspondence between theories and the world could be expressed in terms of isomorphism, or partial isomorphism. This apparently constitutes a more radical departure from Ramsey sentences. However, Ainsworth ([2009]) shows that Newman's objection can be transposed to the semantic view. This is later acknowledged by French ([2014], p. 126). Other authors note that the two conceptions of theories are equivalent in certain respects (unless the semantic view is untenable-see (Halvorson [2012], pp. 203-5)). I will consider that Melia and Saatsi's analysis can be transposed to the semantic view.

${ }^{4}$ It could be associated with transduction, which, according to McGuire ([1970]), is a mode of inference that played a role in Newton's atomism. It amounts to extending characteristics of observable objects to hypothetical, unobservable objects
} 
The most promising option, according to Melia and Saatsi, is to incorporate non-extensional logic, such as modal logic, to express the fact that theoretical relations express nomological relations rather than mere universal regularities. They claim (but do not prove) that this would block the theorem supporting Newman's objection. I think that this is not true, as I will now explain.

\section{Which Modal Relations are Retained in Theory Change?}

I claim that modal logic is not sufficient to block Newman's objection: the position we reach by expressing the content of our theories with modal logic is not structural realism, but modal empiricism. Indeed, it is well known that one can mimic modal logic in extensional logic by quantifying over possible worlds. If we follow Melia and Saatsi's suggestion, we can express the cognitive content of the theory as follows, where the $w_{i}$ are possible worlds:

$\left(\exists t_{1}, t_{2}, \ldots, t_{m}\right)\left(\forall w_{1}, w_{2}, \ldots, w_{p}\right) \prod\left(O_{1}, O_{2}, \ldots, O_{n}, t_{1}, t_{2}, \ldots, t_{m}, \ldots, w_{1}, w_{2}, \ldots, w_{p}\right)$

Perhaps the theory would also have existential quantifiers on possible worlds, not just universal quantifiers, to express possibilities. Perhaps quantifiers would be embedded into its formulation rather than appear at the beginning. However this does not matter for the sake of the argument. In any case, this Ramsey sentence is not formally different from the previous one, and there is no reason that the same theorem would not apply. The conclusion of the theorem would be slightly different though, since possible worlds are interpreted objects that should not be ramseyfied. The conclusion would be the following: the modal Ramsey sentence of a theory is true if and only if all the empirical consequences of the theory are true in all possible worlds, and if there are enough objects in possible worlds to bear the structure of the theory ${ }^{5}$. In sum, this version of the Ramsey sentence merely says that our theory is empirically adequate in all nomologically possible worlds, plus a cardinal claim.

This formulation does not say how possible worlds should be interpreted metaphysically speaking. A minima, we are talking about natural (or nomological) necessity, not conceptual or logical necessity, but modal relations could be causal

\footnotetext{
${ }^{5}$ I will not engage here in the debate between possibilism and actualism, that is, if we should quantify over the same objects or different objects in all possible worlds.
} 
relations, or primitive laws, or relations between universals. Or perhaps nomologically possible worlds are just as concrete as the actual world. In all these cases, possible world semantic can be considered appropriate as a formal tool. Therefore, the conclusion we reach is neutral with regards to the metaphysics of modalities. I will return below to the question of the interpretation of modalities, and their compatibility with an empiricist stance.

At this point, the question is the following: should a structural realist be satisfied with such an account? Is it enough to get 'real' relations? Ladyman ([2004]) suggests that a version of empiricism committed to natural modalities would be nothing but SR. However, Ladyman does not really justify this claim, and I will argue that this is not the case.

The answer depends on what criteria we use: what do we mean by 'real' relations? On a first approach, we could stick to the argument that SR purports to answer. Then we can claim that this solution does the job if (i) modal relations survive theory change and (ii) they are sufficient to explain the empirical success of theories. Point (ii) is crucial to know if modal SR is distinct from modal empiricism, since one of the characteristic differences between realism and empiricism is that the latter does not pretend to explain empirical success while realism, including SR in its different guises, does (see for example Ladyman and Ross [2007], p. 79). However, in order to tackle this question, we must first examine point (i): whether modal relations survive theory change (and what kinds of modal relations do).

There are intuitive reasons to believe that (i) is true. If anything is retained in theory change, it is what Duhem ([1906]) called 'experimental laws'. Theories of light have changed several times since the $17^{\text {th }}$ century, but they all had to account for light reflection and refraction: they just embraced more and more phenomena with time (diffraction, interference, ...). Now assuming that such experimental laws express constraints of necessity in the phenomena they describe apparently does nothing to alter this continuity. At most new theories will restrict the domain to which the old theory is applicable, so that the constraints of necessity between observable phenomena expressed are only approximate (for example, old theories of light neglect magnetic influences, and are only valid when there is no magnetic field). But if the new, wider range constraints are considered physically necessary, then one could think that the old, narrower constraints are physically necessary too, at least restricted to their domain of application.

All this seems intuitively true. However, it does not go without saying. Let us start from the premise that a new, wide-range constraint on phenomena is a matter of nomological necessity, and that it entails some constraint between phenomena 
$A$ and $B$ in the narrow context $C$ ( $A$ could be the fact that there is ancident ray, $B$ the fact that there is a reflected ray with the same angle, and $C$ the fact that there is no magnetic field). This can be expressed as follows, with modal operators indicating nomological necessity:

$$
\square(C \rightarrow(A \rightarrow B))
$$

However, it is not possible, from this premise, to deduce the following:

$$
C \rightarrow \square(A \rightarrow B)
$$

This could only be deduced if $C$ was necessary, but unless all facts are necessary, which would trivialize our position, we cannot assume it: the fact that there is no magnetic field at some place in the universe is not necessary, but contingent.

In other words, if the new theory says: it is necessary that in context $C$, there is a relation between $A$ and $B$, that does not entail that in context $C$, there is a necessary relation between $A$ and $B$. This might not be so much of a problem for an empiricist, who could say that the old theory is still modally adequate if we pragmatically restrict the range of possible worlds to that where $C$ is the case. After all, there are many kinds of relative modalities: for example, technical modalities, which depend on our technical limitations (I will return to the way an empiricist could interpret this kind of relative modality below). But for the structural realist, the fact that there is no necessary relation between $A$ and $B$, but only between $A, B$ and $C$, is problematic because the relation between $A$ and $B$ does not correspond in any sense to the fundamental, modal structure of the world: it only corresponds to the structure of phenomena in situations where $C$ holds; that is to say, it is relative to a context where $C$ is the case. But the fact that $C$ holds in some places, and not others, is contingent, not necessary.

Perhaps the law of the old theory could be seen as an approximation of the new law. Let us say that a modal statement approximates a law of necessity if it is true in 'most possible contexts'. The problem with this strategy is that it requires one to provide a measure on contexts, and it is not obvious how to do so, and whether it makes sense at all. Different contexts could correspond to different models in the new theory, and a theory does not provide a measure on its models. Even granting that it makes sense, it does not seem that the law of an old theory of light that, say, does not take into account magnetic influences, would approximate the law of the new theory, except if we choose an ad-hoc measure on contexts: arguably, there are many more possible configurations of the magnetic 
field where the old law would fail than where the old law would succeed, and in light of theory change, the success of the old law is better accounted for by the fact that we live in places where the magnetic field is weak than by the fact that it could approximate a strict law of necessity. So the relations that are retained in theory change are either contingent, or if modal, they are only so in a relative sense, as are technical modalities, but they are not absolutely modal, nor do they approximate absolutely modal relations.

Maybe this will be more clear with an example. Take Galileo's law of free fall, according to which all falling objects accelerate at $9.8 \mathrm{~m} / \mathrm{s}^{2}$. The law is certainly retained in contemporary physical theories in the sense given by Post's principle of correspondence mentioned above. However, there is no sense in which it is a modal relation pertaining to the fundamental structure of the world: the number ' 9.8 ', although it is part of the mathematical structure of this law, does not appear in contemporary physics, and not even the idea of constant acceleration is retained. Arguably, this law only approximates contemporary ones in a tiny range of possible contexts. This law is rather, in light of new theories, a contingent empirical consequence of the fact that we live on the surface of the earth, and that the earth has a given mass and a given size, but all these contextual aspects, and the way they are involved, are only accessible from the new theories. There is no problem, from an empiricist perspective, in making this kind of relation relative to our epistemic situation in the world, since, after all, an empiricist is often willing to accept that the content of our representation is not absolute but relative to our epistemic position. But postulating a relation of correspondence between this law and the modal structure of the world is more problematic given that it appears to be merely contingent. So it is not true that modal relations are retained in theory change, or at least, not the kind of modal relation a realist would call for.

\section{Are Modal Relations Real?}

Let us now turn to (ii): in what sense would the modal Ramsey sentence constitute an explanation for the empirical success of theories? The idea behind the nomiracle argument is that the fact that the entities postulated by a theory really exist explains the observable phenomena the theory accounts for. But here, rather than positing real entities, it seems that we have merely extended empirical adequacy to other possible worlds, and it is dubious that such an extension could constitute an appropriate explanation (see van Fraassen ([1989], ch. 4.7) for arguments).

Rather than focusing on possible worlds, one could claim that we are postu- 
lating nomological constraints on the phenomena, and that these constraints are the entities that explain observable regularities in the actual world. But in what sense are these nomological constraints 'real entities'? Reality is generally cast in terms of mind-independence. We have two options here: either these relations are 'real' because they are relations between real, mind-independent entities, or they are real 'in themselves', that is, the relations themselves are mind-independent.

The first option corresponds to how epistemic versions of SR, following the lead of Poincaré and Russell, traditionally conceive of real relations. We saw that Newman's objection undermines this view: inaccessible objects play no role in $\mathrm{SR}$, since they only have to be in sufficient number to bear the structure of the theory, so the relevant relations to which SR is committed are actually relations between our observations only. As we saw, the same argument applies to modal SR as well: the relations to which modal SR is committed are only relations between our observations, and inaccessible objects play no particular role.

Perhaps, however, possible observations could count as real entities? One could argue that possible observations are mind-independent, because they are never actually observed. Then modal relations would qualify as relations between real objects. But note that past, future or remote observable phenomena are also mind-independent in this sense. They do not have to be actually observed to exist. However, an empiricist like van Fraassen would accept that our theories are empirically adequate for all observable phenomena in the universe, including phenomena that are not actually observed. The crux here is that mind-independence can be understood in two ways: something is mind-independent either if it does not depend on actually being observed, or if its nature is independent of our conceptualisation of it (but could still correspond to it). We can get the first kind of mind-independence with possible observations, but not the second kind, since possible observations are still conceptualized as observations, and if constructive empiricism is not a brand of realism, the first kind of mind-independence is insufficient for genuine realism.

The second option is to assume that it is modal relations that are real, not what they relate. In other words, the solution is to move to an ontic version of SR, following the lead of Cassirer ([1937]) and Eddington ([1955]) rather than Poincare and Russell, whereby relations are primitive, and the relata are fully determined by the relations in which they take part (or the two are in a co-determination relationship-see (French [2014]) for a review). Cassirer and Eddington conceived of relations as mental entities, but if we are realist, then we can conceive of these relations as primitive ontological entities, and assume a correspondence between the structure of our representations and reality. However, there are problems with 
this move.

A problem that has been discussed at length in the literature is that it is hard to make sense of relations without relata, and still make a distinction between mathematical and physical structure (see for example (Cao [2003]; Psillos [2015])). Defenders of ontic SR want modalities to be the distinctive feature, and at the same time, they assume that the relata of the structure are fully determined by their position in the structure. But how shall we make sense of this? Take the example of a probabilistic law that would say that if $A$ is the case, then $B$ will occur with probability $p$ and $B^{\prime}$ will occur with probability $1-p$, where $A, B$ and $B^{\prime}$ are observable states of affairs. If following ontic SR the modal structure is 'all there is', then something is obviously missing in our ontology: the fact that in such a situation, $B$ actually occurs rather than $B^{\prime}$ is not entailed by the law itself. Perhaps one could reduce this aspect to an indexical component by being a modal realist in the sense of Lewis ([1973]) (here restricted to nomologically possible worlds): $B$ and $B^{\prime}$ both occur in different concrete possible worlds, and the fact that $B$ actually occurs only means that we are located in a world where $B$ occurs. But if this is the case, either the law is no longer primitive: it supervenes on real states of affairs distributed in different possible worlds, or if the nature and distribution of these states of affairs are fully determined by the laws themselves, it's not clear that SR does not collapse into some form of Pythagoreanism: all we are left with is a mathematical structure of concrete, possible worlds.

The problem is reminiscent of Newman's objection analysed in section 2. One could eschew Pythagoreanism by qualifying the structure, and in a sense, 'primitively modal' could be viewed as a mere qualification, but as we saw in section 2 , this qualification will be either too strong to survive theory change, or too weak to escape triviality (see (Ainsworth [2009]) for related arguments).

So as a minimum, we must assume that actual relata exist on a par with a primitive modal structure for this structure to be physical rather than mathematical, and that these actual relata are not determined by the structure. Psillos ([2015]) makes a similar point, noting that laws of nature do not determine the initial conditions of the universe. French ([2014], ch. 10) also acknowledges this point, and talks about existential witnesses (drawing on the distinction between determinable and determinate). But these witnesses cannot be real inaccessible objects, otherwise the position is not informative (for the reasons given by Newman's objection), and if they are our actual observations, it seems that we are back to where we started: the relations we postulate are merely interpreted in relation to our observations, and they are not conceptually mind-independent. At most they would deserve the 'primitively modal' qualification in addition to this empirical interpretation. 
None of this is really new, but the problem is somehow strengthened by our observations from the previous section: modal relations that survive theory change generally become relative to an epistemic context in the process. This shows that the 'primitively modal' qualification is too strong to sustain ontic SR in the face of the pessimistic meta-induction: either the relations that survive theory change are not modal, but contingent, or if they are modal in a restricted sense, they are not primitive, but relative to our specific epistemic position. It does not seem that this qualification can be weakened without thereby undermining the realist component of ontic SR: non-modal relations are merely mathematical, and relatively-modal relations depend too much on our epistemic position to be 'real'. So even if it were possible to make sense of modal relations being real without collapsing into Pythagoreanism, the solution is not viable, because primitive, modal relations do not survive theory change.

\section{Relativity and Fundamentality}

The analysis above applies quite straightforwardly to versions of SR that maintain that our physical theories represent the fundamental modal structure of the world, given that a relatively modal relation $\square R$ cannot count as fundamental in a world where $\square(C \rightarrow R)$ is the case. This applies in particular to French's version, and its repeated emphasis on fundamentality (French [2014], p. 44, p. 114, ch. 10). However, SR, even in its ontic version, is not a monolithic doctrine, and one could wonder whether less metaphysically inclined versions could accommodate relative modalities. ${ }^{6}$

According to Ladyman and Ross ([2007]), the modal relations science describes need not be fundamental. In particular, the 'real patterns' discovered by special sciences are specific to certain domains or scales and are relative to ("projectible under') perspectives (Ladyman and Ross [2007], ch. 4.4). Ladyman and Ross go as far as claiming that they can make sense of relative necessity (Ladyman and Ross [2007], p. 288). This seems at odds with our contention that modal relations that are relative to our epistemic context cannot count as real.

Note, however, that the relativity invoked by Ladyman and Ross is not enrolled in discussions on theory change, but rather on scales and fundamentality. In their account, the perspective to which modal relations are relative can be specified by 'locators'. This might be fine for the prospect of understanding the role of

\footnotetext{
${ }^{6} \mathrm{I}$ am thankful to anonymous referees for raising this point.
} 
special sciences, but in the context of theory change, the relevant context is only accessible in light of a new theory, and so we are in no position to claim that contemporary theories are structurally true relative to a specific context (at least not without alluding to our epistemic position).

Moreover, the problem we are confronted with is not so much a problem of fundamentality. The notion of locators Ladyman and Ross employ seems inappropriate for our purpose. The notion is apparently extensional (Ladyman and Ross [2007], ch. 2.3.4). It amounts to limiting the domain of a theory to part of the actual universe (the parthood relation involved need not be spatio-temporal: it could be, for example, 'at a certain scale', or 'for a particular set of actual phenomena'). However, old and new theories of light alike apply to the same type of phenomena, at the same scale, and as we have seen in section 3, limiting the domain of a theory to parts of the actual universe where $C$ is the case will not make its modal statements true, unless $C$ is necessarily the case in those parts of the universe. So for example, limiting the domain of an old theory of light to parts of the universe where there is no magnetic field will not make its laws true as a matter of necessity, insofar as there could have been a strong magnetic field in those places. In sum, the appropriate limitation for our modal statements is not extensional, but intensional: it is a limitation in terms of possible contexts, where some background conditions that are not specified by the theory must hold in all relevant possibilities.

Finally, note that even though Ladyman and Ross assume that special sciences describe real patterns that are only present at certain scales, they entertain a more ambitious stance towards physics. According to them, fundamental physics discovers structures of a 'higher level of necessity'. It is responsible for supporting counterfactuals across the entire actual universe: the residual relativity of modal statements is relativity to contingent 'structural facts about the whole universe', such as its initial conditions. This is why fundamental physics 'gives the modal structure of the world' (Ladyman and Ross [2007], p. 288).

Such a stance seems necessary for SR to qualify as a version of realism. To that effect, Ladyman and Ross ([2007], p. 99)'s claim that 'ontic structural realism ought to be understood as modal structural empiricism' is instructive. Why classify this position as a brand of realism? For them, '[modal empiricism] is a form of structural realism because according to it the theoretical structure of scientific theories represents the modal structure of reality' (Ladyman and Ross [2007], p. 111) (I will take the faithfulness of this representation to be implicit here). What a faithful representation is, and how it latches onto reality, can be understood in different ways in a structuralist context (see French [2014], ch. 5.10), but one 
would expect that a notion of truth (perhaps partial or approximate truth) that is not epistemically constrained, such as correspondence truth, would be involved at some point. ${ }^{7}$ This should be the case even within a fallibilist approach, wherein SR proponents would grant that theoretical modal statements are revisable and that contemporary theories of fundamental physics are not at the stage of composing a 'grand unified theory' (as exemplified by French [2014], p. 163). They should assume that contemporary theories still approximately capture part of the modal structure of reality.

Yet as we have seen here and in section 3, there is no sense in which a statement $\square R$ could strictly, partly or approximately correspond to a modal structure described by $\square(C \rightarrow R)$. Furthermore, qualifying the relations as 'relatively modal' without specifying the background conditions to which they are relative could lead to triviality in the context of Newman's objection. In light of this, we should assume, at most, that our theories capture the modal structure of observations that are accessible from our epistemic context. Let us call the corresponding position modal empiricism (ME). This ineliminable reference to an epistemic context that we are in no position to specify any further until a new theory arrives is precisely what makes ME a version of empiricism rather than realism: only an epistemically constrained notion of truth, such as pragmatic truth, will account for it.

In sum, Ladyman and Ross's version of ontic structural realism might be as close to ME as SR can get, but their contention that physics gives us the modal structure of the world makes it a distinct position, and unless they provide an account of partial or approximate truth for relative modalities that solves the problems mentioned here, their position cannot be sustained in the face of the pessimistic meta-induction.

\section{Is Modal Empiricism Really Empiricism?}

Our conclusion so far is that Newman's objection also applies to the modal versions of SR: modal SR, if it is to survive theory change, collapses into a version of empiricism, albeit a modal one. Still, one could remain unconvinced that ME is really empiricism. Empiricists are generally suspicious about natural modalities, which they generally consider part of an 'inflationary metaphysics' (see for

\footnotetext{
${ }^{7}$ Arguably, an epistemically constrained notion of truth, such as pragmatic truth, is incompatible with realism. For example, Ladyman and Ross take a pragmatic stance towards individual objects, the existence of which they deny.
} 
example van Fraassen [1989]). This is certainly true if modalities are posited to explain observable regularities. But one could well consider that ME does not explain anything, but merely extends empirical adequacy to possible worlds (or, as I will suggest below, to possible situations).

As emphasized above, the difference between realism and empiricism might be best expressed in terms of correspondence truth. The realist assumes that our representations correspond to a mind-independent reality, while the empiricist merely assumes that they 'save the phenomena'. If we accept this way of framing the distinction, then there is a sense in which modalities can be regimented into an empiricist framework. The way to do this is to translate modal statements not in terms of a direct correspondence to nomological relations, but into warranted expectations about our future experiences (such as: if we were to do $A$, we would observe $B$ ). In sum, the idea is to apply a pragmatic theory of truth to modalities rather than a correspondence theory of truth. After all, Peirce, who introduced pragmatism, was committed to the existence of necessity in the world (Peirce was only a self-proclaimed realist in a weak sense, refusing the correspondence theory of truth), and if Peirce's philosophy is consistent, there should not be any incompatibility between the two.

A pragmatic evaluation of modal relations is available to us so long as the relata of these relations are not inaccessible objects, but observations, which is the case here. And if, as I have argued, modal relations must be considered relative to an epistemic context in order to survive theory change, this is the only interpretation available to us: there is no sense in which any modal relation entailed by our theories would correspond to a nomological constraint independent of our epistemic position. Indeed, according to $\mathrm{ME}$, it can be true, for all practical purposes, that if we had thrown an object, it would have accelerated at $9.8 \mathrm{~m} / \mathrm{s}^{2}$ towards the ground. But this modal statement is only pragmatically true because we consider a certain epistemic context to be fixed (the fact that we are here, on earth). It does not correspond to any absolute necessity, not even approximately so. The earth could lose part of its mass following a cosmic disaster, which would make this statement false. Yet this is still a modal statement, and it can still be pragmatically true, relative to a background context held fixed.

A nice framework for analysing modal statements pragmatically is a possible situations semantic, where situations are partial, rather than complete, worlds (Kratzer [2008]). The idea is to conceive of possible situations as alternative ways actual situations could be, considering variations in some accessible characteristics of situations, 'all else being equal'. The extent of conceivable possibilities can be apprehended in terms of possible values for measurable properties (given the 
precision of our apparatus). The modal empiricist would assume that only some of these conceivable possibilities are nomologically possible, and that it is a matter of empirical inquiry to know which ones are. This way, implicit background conditions and contextual aspects (for example, the fact that we are here, on earth, that the earth has a given size, ...) can be held fixed in the evaluation of modal statements, by directly referring to actual situations of reference, even though these background conditions cannot be listed or even known exhaustively. This context will take the form of unspecified intensional constraints on the range of possibilities that are accessible to us given our epistemic position at one point in time. This is the way relative modal statements can be captured in this framework: the constraints on possibilities that the modal empiricist assumes are only valid relative to these unspecified background conditions, and we need not assume that these background conditions are plausible, or correspond to 'most possible contexts': they are pragmatically held fixed by fiat. I will adopt this framework from now on.

There must certainly be some modal constraints in the world for our expectations to be fulfilled in all possible circumstances, even if they are restricted to a context. A modal empiricist must assume that there are possibilities in the world and constraints on these possibilities (I will return to this aspect below). However, by assuming that a modal relation obtains pragmatically, one is not speculating on the metaphysical nature of the constraints involved, nor is one claiming that they correspond absolutely to the modal relation under consideration. A pragmatic evaluation of a modal statement in terms of observational expectations, even though it implies that there are constraints on possibilities in the world, does not constitute an explanation of any sort for one's specific expectations in the situation at hand. It is a mere statement of these expectations. Therefore, being committed to the modal Ramsey sentence of a theory does not mean being a structural realist, pace Ladyman.

The fact that our expectations are warranted (that they are not mere wishes) is however essential for the truth of the corresponding modal statement, so let us say a little bit more on the question of justification.

Another way to characterize the contrast between empiricism and realism is methodological. Correspondence truth introduces a gap between truth and our capacity to know that something is true from experience alone. Realists are generally willing to fill this gap by resorting to non-empirical criteria of justification, such as explanatory power, simplicity and fruitfulness. These non-empirical crite- 
ria can be subsumed under abduction ${ }^{8}$, or inference to the best explanation, which is involved in the no-miracle argument (that is, non-empirical criteria are constitutive of a good explanation). By contrast, empiricists would view non-empirical criteria as pragmatic or heuristic devices. They would at most assume that induction is warranted, and therefore remain agnostic about the truth of our theories.

In a possible situation framework, if one assumes that actual, experienced situations are a representative sample of a range of possible situations (alternative ways actual situations could be), ME does not need abduction for its justification, but only induction: an induction on possible situations and configurations, of which observed ones are a representative sample. Having seen all objects in free fall accelerate around us at a certain rate, the modal empiricist is justified in believing that all objects would accelerate at the same rate if they were in free fall, whatever their initial position or speed (note that this is not an attempt to explain a regularity, but a mere induction). Extending her inquiry to different domains, she may realize that this law is not universally true: objects do not accelerate at the same rate at higher altitudes. An abductive inference towards the existence of a modal relation that would correspond to the initial acceleration (and explain it) would thus be invalidated. However, the original modal statement, once restricted to a certain context, is still justified by induction.

Note that this fits quite well with scientific practice, at least intuitively: scientists usually test their theories in various configurations, acting on relevant parameters, as if they were proceeding to an induction on all possible values for these parameters. They also contrast alternative hypotheses by implementing configurations where these hypotheses would make different predictions. This can be viewed as an inductive process on possibilities, and not actualities, since the prior assumption that a configuration actually occurs or not in the universe seems irrelevant to their motivation to implement it. Intuitively, only the fact that it could occur, and that they want to know what would happen in that case (which hypothesis would be confirmed), seems relevant.

So far, we have been talking about specific constraints of necessity, but what about the more general assumption that there are possibilities in the world? This assumption is required for our inductive process, but isn't it at odds with empiricism?

There is an intuitive sense in which this assumption is part of our experience. Some cognitive theorists claim that possibilities of action are part of our phe-

\footnotetext{
${ }^{8}$ Note that Peirce, who introduced the term, did not view abduction as a principle of justification, contrarily to contemporary realists, but rather as a principle of discovery (Nyrup [2015]).
} 
nomenology (Gibson [1986]; Nanay [2011]). The possibility of inferring metaphysical assumptions from our phenomenology could be challenged, but in any case, no epistemological position comes completely devoid of metaphysical commitments. Van Fraassen ([2002]) is well aware that an epistemological position cannot be justified by experience alone, and casts empiricism as a 'stance' for this very reason. ME might be a little more adventurous than constructive empiricism in this respect, but insofar as the specific modal relations that appear in the modal Ramsey sentence of a theory do not count as explanations, but as extensions of universal regularities, insofar as they are justified by induction rather than by abduction, and insofar as the modal empiricist does not assume that they must approximately capture the modal structure of reality, ME does not qualify as a brand of scientific realism.

\section{Could Modal Empiricism be the Best of Both Worlds?}

In this article, I have argued that modal relations do not survive theory change, contrarily to what SR assumes, unless they are considered relative to a contingent epistemic context, which makes them unfit to sustain a realist position. I have also argued that, nevertheless, modal relations that are relative to an epistemic context can be interpreted pragmatically in full compatibility with empiricism and with an inductive epistemology. SR thus either collapses into ME, or falls prey to the pessimistic meta-induction. To conclude, let us examine briefly how ME fares in the debate on scientific realism.

A modal empiricist should not worry too much about the pessimistic metainduction since, as we have seen, the modal relations she is committed to survive theory change. Past theories are still modally adequate in a restricted domain of experience (in a range of possible situations) and science advances by accumulating empirical knowledge. Scientific progress can be viewed as an extension of the range of epistemic contexts available to us, which does not mean that we could be free of any epistemic context at any point in time, or that we could claim to have access to 'most possible epistemic contexts', whatever that means. Now, should the modal empiricist worry about the no-miracle argument?

The modal empiricist cannot claim to explain the empirical success of theories: she is merely extending this success to possible situations. This is why ME is not a version of realism. However, ME might have the resources to answer the no-miracle argument in a different way. Indeed, we can expect that a modally adequate theory will be empirically adequate in situations it was not designed 
to account for if these situations are only considered possible in our epistemic context prior to their occurrence, in the same way that a constructive empiricist would readily extend empirical adequacy to hitherto unobserved phenomena (but perhaps the constructive empiricist would have to remain agnostic about hitherto unobserved configurations, insofar as she does not know if they are actual or not prior to their occurrence). And in the case of a new theory that has not yet been properly tested, successful novel predictions merely confirm that the theory can be extended to a new range of phenomena; yet promising theories can fail in this respect, as has happened in the past. So ME could be the best position of compromise in the debate on scientific realism: not because it would explain the empirical success of theories, but because if the aim of science is to produce modally adequate theories, then the success of novel predictions is no miracle: it is either the first step of an induction on possible situations that will confirm a theory's modal adequacy, at least in a certain context, or, in the case of theories that have already been tested, it is a mere consequence of their modal adequacy, and it is full part of our expectations. However, the eventual failure of these theories when we extend the range of experimental contexts, and the need for a new theory to address new experimental results, should not come as a surprise either.

All this is only a proposal at this stage. Developing the idea further is beyond the scope of this paper. I merely want to stress that in one sense, structural realists could be right that one can have the best of both worlds (to take Worrall's words) by focusing on the modal structure of theories. Their only mistake is to classify their position as a version of scientific realism, while if it is to survive theory change, it can be nothing but empiricism.

\section{References}

Ainsworth, P. [2009]: 'Newman's Objection', British Journal for the Philosophy of Science, 60(1), pp. 135-171.

Bueno, O. [2008]: 'Structural Realism, Scientific Change, and Partial Structures', Studia Logica, 89(2), pp. 213-235.

Cao, T. Y. [2003]: 'Can We Dissolve Physical Entities into Mathematical Structures?', Synthese, 136(1), pp. 57-71.

Cassirer, E. [1937]: Determinismus Und Indeterminismus in der Modernen Physik 
Historische Und Systematische Studien Zum Kausalproblem, Elanders Boktryckeri Aktiebolag.

Churchland, P. and Hooker, C. [1985]: Images of Science: Essays on Realism and Empiricism, University of Chicago Press.

Cruse, P. [2005]: 'Ramsey Sentences, Structural Realism and Trivial Realization', Studies in History and Philosophy of Science Part A, 36(3), pp. 557-576.

Demopoulos, W. and Friedman, M. [1985]: 'Bertrand Russell's the Analysis of Matter: Its Historical Context and Contemporary Interest', Philosophy of Science, 52(4), pp. 621-639.

Duhem, P. [1906]: La théorie physique: son objet, et sa structure, Chevalier \& Rivière.

Eddington, A. [1955]: The Nature of the Physical World, volume 39, London, Dent.

French, S. [1998]: 'On the Whithering Away of Physical Objects', In Interpreting Bodies, Princeton University Press, pp. 93-113.

French, S. [2014]: The Structure of the World: Metaphysics and Representation, Oup Oxford.

French, S. and Ladyman, J. [2003]: 'Remodelling Structural Realism: Quantum Physics and the Metaphysics of Structure', Synthese, 136(1), pp. 31-56.

Gibson, J. [1986]: The Ecological Approach To Visual Perception, Routledge, $<$ http://www.tandfebooks.com/isbn/9780203767764>.

Halvorson, H. [2012]: 'What Scientific Theories Could Not Be', Philosophy of Science, 79(2), pp. 183-206.

Hanrahan, R. [2009]: 'Consciousness and Modal Empiricism', Philosophia, 37(2), pp. 281-306.

Kratzer, A. [2008]: 'Situations in Natural Language Semantics', In A. Kratzer (ed.), Stanford Encyclopedia of Philosophy.

Ladyman, J. [1998]: 'What is Structural Realism?', Studies in History and Philosophy of Science, 29, pp. 409-424. 
Ladyman, J. [2004]: 'Constructive Empiricism and Modal Metaphysics: A Reply to Monton and Van Fraassen', British Journal for the Philosophy of Science, 55(4), pp. 755-765.

Ladyman, J. and Ross, D. [2007]: Every Thing Must Go: Metaphysics Naturalized, Oxford University Press.

Laudan, L. [1981]: 'A Confutation of Convergent Realism', Philosophy of Science, 48(1), pp. 19-49.

Lewis, D. [1973]: Counterfactuals, Blackwell Publishers.

Maxwell, G. [1971]: 'Structural Realism and the Meaning of Theoretical Terms', Minnesota Studies in the Philosophy of Science, 4, pp. 181-192.

McGuire, J. [1970]: 'Atoms and the 'Analogy of Nature': Newton's third rule of philosophizing', Studies in History and Philosophy of Science Part A, 1(1), pp. $3-58$.

Melia, J. and Saatsi, J. [2006]: 'Ramseyfication and Theoretical Content', British Journal for the Philosophy of Science, 57(3), pp. 561-585.

Nanay, B. [2011]: 'Do we See Apples as Edible?', Pacific Philosophical Quarterly, 92(3), pp. 305-322.

Newman, M. [1928]: 'Mr. Russell's Causal Theory of Perception', Mind, 5(146), pp. 26-43.

Newman, M. [2005]: 'Ramsey Sentence Realism as an Answer to the Pessimistic Meta-Induction', Philosophy of Science, 72(5), pp. 1373-1384.

Nyrup, R. [2015]: 'How Explanatory Reasoning Justifies Pursuit: A Peircean View of IBE', Philosophy of Science, 82(5), pp. 749-760.

Papineau, D. [1996]: The Philosophy of Science, Oxford University Press.

Pashby, T. [2012]: 'Dirac's Prediction of the Positron: A Case Study for the Current Realism Debate', Perspectives on Science, 20(4), pp. 440-475.

Post, H. [1971]: 'Correspondence, Invariance and Heuristics: In Praise of Conservative Induction', Studies in History and Philosophy of Science Part A, 2(3), pp. 213-255. 
Psillos, S. [1995]: 'Is Structural Realism the Best of Both Worlds?', Dialectica, 49(1), pp. 15-46.

Psillos, S. [1999]: Scientific Realism: How Science Tracks Truth, Routledge.

Psillos, S. [2015]: 'Broken Structuralism: Steven French: The Structure of the World: Metaphysics and Representation.', Metascience, <http://link.springer.com/10.1007/s11016-015-0030-0>.

Putnam, H. [1975]: Mathematics, Matter, and Method, Cambridge University Press.

Redhead, M. [2001]: 'The Intelligibility of the Universe', Royal Institute of Philosophy Supplement, 48, pp. 73-90.

Roca-Royes, S. [2007]: 'Mind-Independence and Modal Empiricism', In C. Penco (ed.), Proceedings of the 4th Latin Meeting in Analytic Philosophy, 117-135.

Russell, B. [1927]: The Analysis of Matter, London: Kegan Paul.

Stanford, K. [2003]: 'Pyrrhic Victories for Scientific Realism', Journal of Philosophy, 100(11), pp. 553-572.

van Fraassen, B. [1980]: The Scientific Image, Oxford University Press.

van Fraassen, B. [1989]: Laws and Symmetry, volume 102, Oxford University Press.

van Fraassen, B. [2002]: The Empirical Stance, The Terry Lectures, New Haven, CT: Yale University Press.

Votsis, I. [2004]: The Epistemological Status of Scientific Theories: An Investigation of the Structural Realist Account.

Worrall, J. [1989]: 'Structural Realism: The Best of Both Worlds?', Dialectica, 43(1-2), pp. 99-124.

Zahar, E. [2004]: 'Ramseyfication and Structural Realism', Theoria, 19(1), pp. 5-30. 